\title{
B-Vitamin Therapy for Kidney Transplant Recipients Lowers Homocysteine and Improves Selective Cognitive Outcomes in the Randomized FAVORIT Ancillary Cognitive Trial
}

\author{
T.M. Scott ${ }^{1}$, G. Rogers' ${ }^{2}$ D.E. Weiner ${ }^{3}$, K. Livingston ${ }^{2}$, J. Selhub ${ }^{4}$, P.F. Jacques ${ }^{2}$, I.H. Rosenberg', \\ A.M. Troen ${ }^{1,4,5}$ for the FACT Study Investigators ${ }^{t}$
} 1. Neuroscience and Aging Laboratory, Jean Mayer USDA Human Nutrition Research Center on Aging at Tufts University, Boston MA, USA; 2. Nutritional
Epidemiology Program, Jean Mayer USDA Human Nutrition Research Center on Aging at Tufts University, Boston MA, USA; 3. Division of Nephrology, Tufts Medical
Center and Tufts School of Medicine, Boston MA, USA; 4. Vitamin Metabolism Laboratory, Jean Mayer USDA Human Nutrition Research Center on Aging at Tufts
University, Boston MA, USA; 5. Institute of Biochemistry, Food Science and Nutrition, The Robert H. Smith Faculty of Agriculture, Food and Environment, The Hebrew
University of Jerusalem, Rehovot, Israel. t. See appendix A in online version.

Corresponding Author: Prof. Aron M. Troen, Director, Nutrition and Brain Health Laboratory, Institute of Biochemistry, Food Science and Nutrition, The Robert H. Smith Faculty of Agriculture, Food and Environment, The Hebrew University of Jerusalem, P.O. Box 12, Rehovot 76100, Israel, Phone ++972-54-8820420, E-mail: aron.troen@mail. huji.ac.il

\section{Abstract}

BACKGROUND: Objectives: Elevated plasma total homocysteine (tHcy) is associated with increased risk of cardiovascular disease, stroke and dementia. Results of clinical trials using B-vitamins to reduce the cognitive risks attributed to tHcy have been inconsistent. The high prevalence of both hyperhomocysteinemia and cognitive impairment among kidney transplant recipients makes them an important population in which to evaluate the effect of lowering homocysteine on cognitive function. We therefore evaluated whether B-vitamin therapy to lower tHcy would prevent cognitive-decline in a cohort of stable kidney transplant recipients.

DESIGN: The study was a longitudinal ancillary of the FAVORIT trial, a randomized, placebo-controlled multi-site trial of high-dose B vitamins to reduce cardiovascular and cerebrovascular events in clinically stable kidney transplant recipients with elevated tHcy.

PARTICIPANTS: 584 participants from 18 sites across North America.

INTERVENTION: The intervention consisted of a daily multivitamin containing high-doses of folate $(5.0 \mathrm{mg})$, vitamin B12 (1.0 mg) and vitamin B6 (50 mg). The placebo consisted of a daily multi-vitamin containing no folate and recommended daily allowances of vitamins B12 and B6 (0 mg folate; $2.0 \mu \mathrm{g}$ vitamin B12; $1.4 \mathrm{mg}$ vitamin B6).

MEASUREMENTS: Annual neuropsychological assessment for up to 5 years (mean 3.3 years) using a standardized test battery. Efficacy was analyzed on an intention-to-treat basis using endof-trial data. Subgroup analyses included stratification for baseline plasma B-vitamin and tHcy concentrations.

Results: At baseline, cognitive impairment was common with $61 \%$ of participants falling more than one standard deviation below published norms for at least one cognitive test. Fewer than $1 \%$ of participants had insufficient plasma folate $<5 \mathrm{ng} /$ $\mathrm{ml}$ or vitamin B12 < $148 \mathrm{pmol} / \mathrm{L}$. However, $44.6 \%$ had plasma B6 concentrations $<30 \mathrm{nmol} / \mathrm{L}$. At follow-up, processing speed and memory scores were modestly but significantly better in the $B$-vitamin supplement group than in controls $(\mathrm{p} \leq 0.05)$. There was no interaction between baseline tHcy, B-vitamin status and treatment on the cognitive outcomes.
CONCLUSIONS: High-dose B-vitamin supplementation provided modest cognitive benefit for kidney transplant recipients with elevated baseline tHcy. Since nearly all participants were folate and vitamin B12 sufficient at baseline, the potential cognitive benefits of folate and B12 supplementation in individuals with poor B-vitamin status remains to be determined.

Key words: Folate, B-vitamins, homocysteine, cognition, kidney transplant.

\section{Introduction}

$\mathbf{M}$ ild to moderately elevated plasma total homocysteine (tHcy) concentrations are associated with impaired cognitive function in older adults, and with significantly increased risk of cardiovascular disease, cerebrovascular disease, cognitive decline and dementia (1-7). These associations are thought to result from homocysteine-induced vascular damage and from altered homocysteine metabolism and methylation activity in the periphery and brain.

Because the B-vitamins folate, B12 and B6 are important determinants of homocysteine metabolism and supplementation with these vitamins can lower circulating tHcy, B-vitamin therapy to lower tHcy has been studied in a variety of settings as a way to reduce the cerebrovascular and cognitive risks that are attributed to homocysteine. The results of these trials have been mixed and their collective interpretation remains controversial, due to heterogeneity in design, study limitations and other considerations (8-11). Several trials in healthy older adults (12), elders with vascular disease (13), individuals with mild cognitive impairment (14), elders with mild vitamin B12 deficiency (15), and 
dementia (16), found that B-vitamin treatment did not benefit cognition despite lowering tHcy. Other trials, however, find better cognitive outcomes following treatment with high-dose $\mathrm{B}$ vitamins compared to placebo, primarily in individuals at-risk of cognitive decline with elevated plasma tHcy concentrations at baseline (17-19). Notably, the recent VITACOG trial tested the effect of a daily dose of $0.8 \mathrm{mg}$ folic acid, 0.5 $\mathrm{mg}$ vitamin $\mathrm{B} 12$ and $20 \mathrm{mg}$ vitamin B6 versus placebo on brain atrophy over 2 years, in individuals who met the clinical criteria for Mild Cognitive Impairment (MCI). The results showed that relative to placebo, B vitamin treatment stabilized executive function independently of tHcy and slowed both brain atrophy and decline in the Mini Mental State Examination (MMSE) and semantic and episodic memory in those participants who had a baseline plasma tHcy concentration above $11.3 \mu \mathrm{mol} / \mathrm{L}$, but had no effect in those with concentrations below that level (19-21).

Both hyperhomocysteinemia and cognitive impairment are common in individuals with chronic kidney disease (22-24). Although tHcy concentrations fall modestly after kidney transplantation, elevations of tHcy typically persist, remaining in the range associated with increased risk of cerebrovascular disease and cognitive decline in the general population $(25,26)$. In contrast to findings among dialysis patients, tHcy concentrations can be further lowered among stable transplant recipients through high-dose B-vitamin therapy $(27,28)$. Since kidney transplant recipients have a high prevalence of hyperhomocysteinemia as well as a high risk of cognitive impairment, they are a useful test population to evaluate the effect of B-vitamin therapy to lower tHcy on cognitive function.

In this context, the present study was designed to determine whether B-vitamin therapy would prevent cognitive decline in patients with hyperhomocysteinemia who had received a kidney-transplant: FACT (the FAVORIT Ancillary Cognitive Trial) (29), was a longitudinal ancillary study of the Folic Acid for Vascular Outcome Reduction in Transplantation trial (FAVORIT), a multisite, randomized, double-blind, placebo-controlled clinical trial that examined the effects of treatment with B-vitamins to lower tHcy on cardiovascular outcomes in kidney transplant recipients with mild to moderately elevated tHcy.

Although B-vitamin therapy did not improve cardiovascular endpoints in FAVORIT despite lowering tHcy (30), the cognitive effects of treatment in this population have not yet been reported.

\section{Participants and Methods}

\section{FAVORIT (parent trial) summary}

The design and results of the parent trial have been previously reported $(30,31)$ and the trial is registered at ClinicalTrials.gov under identifier NCT00064753. Briefly, participants were men and women aged 35 to 75 years with stable kidney function for at least 6 months after transplantation and with elevated tHcy of $\geq 12.0 \mu \mathrm{mol} / \mathrm{L}$ for men or $\geq 11.0 \mu \mathrm{mol} / \mathrm{L}$ for women. The trial enrolled study participants from 30 sites in the USA, Canada and Brazil between August 2002 through January 2007, with follow-up contacts occurring every 6 months and annual clinic visits. Participants randomized to the treatment group received a daily multi-vitamin containing high-doses of folate $(5.0 \mathrm{mg})$, vitamin B12 (1.0 $\mathrm{mg}$ ) and vitamin B6 (50 mg). Those randomized to the placebo group received a daily multi-vitamin containing no folate and doses of vitamins B12 and B6 consistent with recommended daily allowances (folate $0 \mathrm{mg}$; vitamin B12, $2.0 \mu \mathrm{g}$; and vitamin B6, $1.4 \mathrm{mg}$. Mandatory fortification of flour with folic acid was in effect in the participant's countries throughout the trial. The primary outcome of FAVORIT was pooled incident or recurrent cardiovascular disease. The study was terminated early on June 24, 2009 after an interim analysis by the Data Safety and Monitoring Board concluded that the incidence of cardiovascular disease, all-cause mortality, and onset of dialysis-dependent kidney failure did not differ by treatment.

\section{FACT participant enrollment}

FACT was designed to recruit at least 800 subjects for in-person cognitive testing at all 30 of the FAVORIT sites, which was estimated to provide more than adequate power to detect a $5 \%$ difference between treatment and control groups, and with a standard deviation of $25 \%$ of the mean, even with attrition. A subsequent power calculation based on the collected data showed sufficient power to detect a $6.5 \%$ difference between treatment and control groups. Of the 30 FAVORIT sites, 18 sites in North America conducted in-person cognitive testing for the ancillary study (for participating sites Appendix A)\#. FAVORIT participants at these sites were eligible to enroll in FACT, with exclusions for visual or hearing impairment substantial enough to hinder performance on cognitive testing. Since the initiation of the parent FAVORIT trial predated the initiation of FACT by more than a year, FAVORIT participants were eligible for FACT at either the time of their randomization into FAVORIT or the time of an annual FAVORIT followup visit. Participants were followed annually for the duration of the parent trial. The study received approval from the institutional review or ethics boards of all 18 clinical sites and Tufts University. Written informed consent was obtained from all participants.

\section{Cognitive testing}

A battery of well-validated neuropsychological tests was administered in person during participants' 


\begin{tabular}{|c|c|c|c|}
\hline Cognitive Domain & Test & Description & Scoring \\
\hline \multirow[t]{2}{*}{$\begin{array}{l}\text { Executive Function and } \\
\text { Mental Processing Speed }\end{array}$} & Trails A\&B (37) & $\begin{array}{l}\text { "Connect-the-dots" for (A) } \\
\text { consecutive number sequence, } \\
\text { and (B) alternating between } \\
\text { numbers and letters }\end{array}$ & $\begin{array}{l}\text { Number of seconds to } \\
\text { completion, test censored at } 300 \\
\text { seconds }\end{array}$ \\
\hline & Digit Symbol Coding (36) & $\begin{array}{l}\text { Decoding symbols matched to } \\
\text { a digits provided in an answer } \\
\text { key; number copied in } 2 \text { minutes }\end{array}$ & $\begin{array}{l}\text { Number of copied symbols in } \\
\text { two minutes, score range } 0 \text { to } 133\end{array}$ \\
\hline Depression & CES-D (59) & $\begin{array}{l}\text { Rating of depressive symptoms } \\
\text { experience over the past month }\end{array}$ & $\begin{array}{l}\text { Number of symptoms endorsed, } \\
\text { weighted by severity, score } \\
\text { range: } 0 \text { to } 60\end{array}$ \\
\hline
\end{tabular}

FAVORIT clinic visits. These tests were selected to evaluate multiple domains of cognition and mood, including memory, executive function, processing speed and depression (Table 1). Research staff at participating FAVORIT clinics were trained in test administration at bi-annual training sessions held at Tufts University, with on-site sessions occurring as needed to ensure consistent and valid testing procedures. On average, participants were tested twice (range $1-5$ tests), with a mean of 3.3 years on treatment prior to their final testing session. Of the 220 participants who had only one cognitive testing session, most were conducted towards the end of the trial rather than the beginning of the FAVORIT study.

\section{Biomarkers}

Archived baseline blood was assayed for biochemical markers. Plasma tHcy was measured by high-performance liquid chromatography (32). Serum creatinine was determined by kinetic Jaffe assay which was calibrated to the IDMS standard, with estimated glomerular filtration rate (eGFR) calculated using the Chronic Kidney Disease Epidemiology Collaboration (CKD-EPI) equation (33). Plasma folate and vitamin B12 (cyanocobalamin) were measured using the Quantaphase II radioassay kit (Bio-Rad Laboratories, Hercules, CA). Pyridoxal 5'-phosphate (vitamin B6) was determined by the tyrosine decarboxylase apoenzyme method (34).

\section{Covariates}

Most demographic and clinical data were ascertained from the FAVORIT parent trial with additional selfreported demographic data, including duration of transplant and education, collected specifically for FACT. Prior to modeling, we examined distributions of demographic, health-related, and study design variables between the intervention and control groups to identify potentially significant covariates. Statistical models were accordingly adjusted for the following: age at baseline, sex, race, education, hypertension at baseline, diabetes at baseline, duration of transplant, number of testing timepoints, FAVORIT site, and eGFR.

\section{Statistics}

All statistical analyses were performed using SAS software (SAS Institute, version 9.3). Unless otherwise stated, statistical significance refers to 2-sided $p$ values $<0.05$. Intervention efficacy was analyzed on an intention-to-treat basis. We calculated least squares means (lsmeans) and 95\% confidence intervals (95\% CI) for baseline demographic, lifestyle, and healthrelated characteristics across treatment and control groups. For positively skewed data, a natural logarithmic transformation was applied and geometric means presented. Except where otherwise indicated, cognitive test results presented are from end-of-trial data (i.e. the final test scores for each participant). Cognitive differences between treatment and control groups were evaluated by comparing lsmeans and 95\% CI from generalized linear models (PROC GLM). Tobit regressions were used for analyses of the Trails Making tasks, since this outcome is right-censored. The effect of the intervention on tHcy was evaluated by calculating differences between tHcy concentrations before- and 1-year after the intervention began. Interactions terms for treatment effect with both baseline B-vitamin and tHcy 


\begin{tabular}{|c|c|c|}
\hline & Treatment $\mathbf{N}=\mathbf{2 5 0}$ & Control $\mathrm{N}=274$ \\
\hline Age at baseline; yrs & $52.9(51.7,54.1)$ & $52.5(51.4,53.6)$ \\
\hline \multicolumn{3}{|l|}{ Race; \% } \\
\hline White & $75.2(70.1,80.3)$ & $81(76.1,85.9)$ \\
\hline Other & $5.6(3.1,8.1)$ & $3.3(0.9,5.7)$ \\
\hline Education; years & $15.2(14.9,15.6)$ & $15.4(15.1,15.7)$ \\
\hline Current smoker; \% & $14.4(10.3,18.5)$ & $10.1(6.2,14)$ \\
\hline Cadaver transplant; $\%$ & $59.8(53.8,65.9)$ & $62.3(56.5,68.1)$ \\
\hline History of cardiovascular disease; $\%$ & $15.6(10.9,20.3)$ & $18.9(14.4,23.4)$ \\
\hline Hypertension; \% & $90.8(87.3,94.3)$ & $91.6(88.2,95)$ \\
\hline Diabetes; \% & $36.8(30.7,42.9)$ & $42.0(36.2,47.8)$ \\
\hline Estimated GFR ${ }^{1}$ & $45.0(42.9,47.2)$ & $45.6(43.5,47.7)$ \\
\hline tHcy; umol / L & $16.8(16.2,17.4)$ & $16.5(15.9,17.0)$ \\
\hline Vitamin B12; pg/ml ${ }^{1}$ & $511(478,548)$ & $546(512,583)$ \\
\hline$<148 \mathrm{pmol} / \mathrm{L} ; \%$ & $0.4(-0.4,1.2)$ & $0.4(-0.4,1.2)$ \\
\hline Folate; $\mathrm{ng} / \mathrm{ml}^{1}$ & $15.9(14.7,17.2)$ & $15.4(14.4,16.7)$ \\
\hline
\end{tabular}

1. geometric means and $95 \% \mathrm{CI}$; no statistically significant between group differences

status assessed whether participants with lower baseline plasma concentrations of B vitamins (folate, vitamin B12, vitamin B6) or higher baseline tHcy experienced different effects of treatment on cognitive performance compared to those with higher initial B-vitamin or lower tHcy concentrations. All models were adjusted for the covariates listed above.

Raw cognitive test scores were converted to ageadjusted scaled-scores for the Word List Learning, Block Design and Digit Symbol tests $(35,36)$ and were converted to age, sex and education adjusted t-scores for the Trails Making tests (37), in order to calculate the percentage of impaired participants by comparing group $\mathrm{t}$-scores to population-appropriate published normative data. If the mean test score fell one standard deviation or more below the normative mean, then performance on the test was considered impaired (29). Chi square tests were used to determine if the percent of impaired subjects differed by treatment.

Longitudinal changes in cognition were analyzed for the subset of participants recruited into FACT at their FAVORIT baseline visit prior to beginning the trial intervention and for whom follow-up data were available. Change in cognitive test scores was calculated as [(last available test - first available test) / years between tests]. We also used the models described above to assess whether the magnitude of change in tHcy (tHcy measured one year after intervention - tHcy measured at screening) was associated with change in cognitive function. All models were adjusted for baseline cognitive test scores in addition to the covariates listed above. Cognitive function among the subset of participants who were assessed at their FAVORIT baseline visit before treatment began was previously described elsewhere (29). 


\section{Results}

\section{Cohort description}

584 participants were enrolled and followed annually for up to 5.5 years. Figure 1 shows the enrollment and analysis numbers for the study. Only participants who had at least one testing session at least 6 months after the start of their FAVORIT study intervention and no missing covariates were included in analyses. Table 2 presents a summary of the participant descriptive data by treatment group. There were no statistically significant differences in baseline characteristics between treatment arms. Overall, participants were predominantly male $(63 \%)$ and white $(78 \%)$, with a mean age of 52.7 years. They were well educated, with many having attended college $(60.1 \%)$. In addition to elevated tHcy which was selected for by design, the prevalence of other risk factors for cognitive impairment was high: Approximately $17 \%$ had a prior history of cardiovascular disease; $91 \%$ had been diagnosed with hypertension and $40 \%$ with diabetes. Mean baseline plasma tHcy was $16.6 \mu \mathrm{mol} / \mathrm{L}$. Less than $1 \%$ of participants had deficient plasma folate $<$ $4 \mathrm{ng} / \mathrm{ml}$ (38) or vitamin B12 < $148 \mathrm{pmol} / \mathrm{L}$ (39). However, $44.6 \%$ had evidence of vitamin B6 deficiency with plasma concentrations $<30 \mathrm{nmol} / \mathrm{L}(40,41)$.

Figure 1. FACT enrollment, follow-up and analysis flow-chart

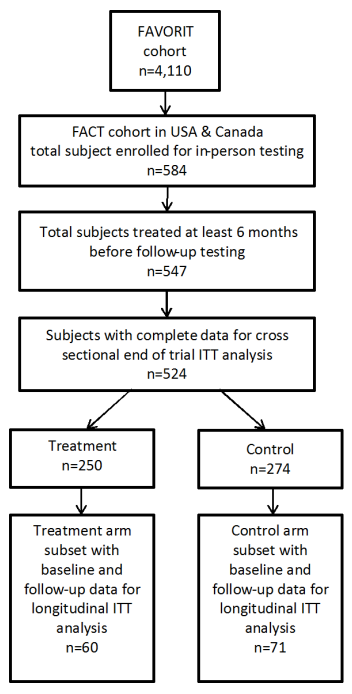

\section{Effect of treatment on tHcy}

Circulating tHcy decreased significantly in both the treatment and control groups from samples taken at screening before the intervention began to those taken one year after the start of intervention. The decrease in tHcy was greater for the treatment group (mean difference $=6.0 \mu \mathrm{mol} / \mathrm{L}$, s.e. $= \pm 0.61, \mathrm{p}<0.0001)$ than for the control group (mean difference $=1.5 \mu \mathrm{mol} / \mathrm{L}$, s.e. $=$ $\pm 0.63 ; \mathrm{p} \leq 0.0001)$. Although B-vitamin supplementation was efficacious in lowering tHcy on average, it failed to normalize tHcy for a large proportion of subjects: $48.5 \%$ of the treatment group and $85.1 \%$ of the control group remained above the cut-points of $>12 \mu \mathrm{mol} / \mathrm{L}$ for men, and $>11 \mu \mathrm{mol} / \mathrm{L}$ for women.

\section{Cognitive Function}

Table 3 gives the cross-sectional adjusted test scores at the end-of-trial, by treatment group. The high-dose vitamin group performed significantly better than the control group on the Digit Symbol Test (52.2 vs. 49.6 $\mathrm{p}=0.05$ ) and Word List Delayed Recall (7.2 vs. 6.7; $\mathrm{p} \leq 0.05$ ). Scores for the other tests and for the CES-D did not differ by group. Cognitive impairment at the end of the trial was common in this kidney-transplant study-population as compared to age-appropriate normative data for the healthy population (Table 4). At the end of the trial, there was a trend $(\mathrm{P}=0.06)$ for a smaller proportion of the B vitamin group (56.4\%) to show overall cognitive impairment than the placebo group (64.6\%). This was significant in the test related to processing speed and attention (31.6\% versus $43.9 \%$ impaired on the Digit Symbol Substitution test; $\mathrm{p}=0.004)$.

Table 3. End-of-trial cross-sectional analyses cognitive testing data

\begin{tabular}{|c|c|c|c|}
\hline \multicolumn{4}{|c|}{ Adjusted Means (95\% confidence intervals) } \\
\hline & $\begin{array}{l}\text { Treatment } \\
(\mathrm{n}=250)\end{array}$ & $\begin{array}{l}\text { Control } \\
(n=274)\end{array}$ & P-value \\
\hline $\begin{array}{l}\text { Word List } \\
\text { Immediate Recall }\end{array}$ & $32.0(30.6,33.5)$ & $31.5(30.0,33.0)$ & 0.30 \\
\hline $\begin{array}{l}\text { Word List Delayed } \\
\text { Recall }\end{array}$ & $7.2(6.6,7.8)$ & $6.7(6.1,7.4)$ & 0.04 \\
\hline Trails A* & $39.1(35.6,42.8)$ & $40.4(36.8,44.4)$ & 0.30 \\
\hline Trails B* & $96.33(86.8,106.9)$ & $98.0(88.2,108.9)$ & 0.64 \\
\hline Digit Symbol & $52.2(48.4,56.1)$ & $49.6(45.7,53.5)$ & 0.05 \\
\hline Block Design & $32.0(29.4,34.5)$ & $31.4(28.8,34.0)$ & 0.51 \\
\hline CES-D ${ }^{* *}$ & $8.96(7.13,11.19$ & $8.93(7.09,11.20)$ & 0.97 \\
\hline
\end{tabular}

Adjusted for age at baseline, sex, race, education, hypertension at baseline, diabetes at baseline, eGFR, time on treatment, number of testing time-points, and FAVORIT site (for North American cohort); ${ }^{*}$ Models for Trails A and B used Tobit regression to censor for failure to complete the task within the allotted 5 minutes (Tobin, 1958) ** Geometric means and 95\% confidence intervals are presented.

\section{Cognitive Decline}

Longitudinal analysis of change in cognition by treatment group was performed for those 131 participants whose initial face-to-face cognitive test was administered prior to the start of their FAVORIT treatment, and who had follow-up cognitive data for a time-point that was a year or later than the start of their treatment (Table $5)$. Contrary to our prediction and despite the high 
prevalence of cerebrovascular risk factors in this cohort (e.g. diabetes, hypertension and hyperhomocysteinemia), we did not observe cognitive decline in this cohort. Indeed the treatment group improved significantly on the Digit Symbol Substitution test compared to the control group (Mean change 2.2 vs. 0.1 respectively, $p=0.03$; Table $5)$. The change in other tests did not differ by group nor was any association found between the magnitude of change in tHcy and the change in any tests of cognitive function (data not shown).

\begin{tabular}{|c|c|c|c|}
\hline Cognitive Test & $\begin{array}{c}\text { Treatment } \\
(n=250)\end{array}$ & $\begin{array}{l}\text { Control } \\
(\mathrm{n}=274)\end{array}$ & $\begin{array}{l}\text { Chi-Square } \\
\text { P-value }\end{array}$ \\
\hline $\begin{array}{l}\text { Word List - Immediate } \\
\text { Recall }^{1}\end{array}$ & 16.5 & 16.8 & 0.92 \\
\hline Word List - Delayed Recall ${ }^{1}$ & 2.4 & 2.6 & 0.91 \\
\hline Trails $\mathrm{A}^{2}$ & 28.5 & 31.4 & 0.47 \\
\hline Trails B ${ }^{2}$ & 30.7 & 33.5 & 0.49 \\
\hline Digit Symbol $^{3}$ & 31.6 & 43.9 & 0.004 \\
\hline Block Design $^{3}$ & 8.0 & 7.7 & 0.89 \\
\hline Any impairment & 56.4 & 64.6 & 0.06 \\
\hline
\end{tabular}

Normative data are from 1. (35); 2. (37); 3. (36); Impairment was defined as a score $\geq 1$ standard deviation below age 1,3 or age, sex, and education 2 specific normative mean scores.

Table 5. Longitudinal change in cognitive scores*

\begin{tabular}{|l|c|c|c|}
\hline Cognitive Test & $\begin{array}{c}\text { Treatment } \\
(\mathbf{n = 6 0 )}\end{array}$ & $\begin{array}{c}\text { Control } \\
(\mathbf{n = 7 1 )}\end{array}$ & P-value \\
\hline $\begin{array}{l}\text { Word List } \\
\text { Immediate Recall }\end{array}$ & $2.3(0.9,3.7)$ & $2.6(1.1,4.0)$ & 0.22 \\
\hline $\begin{array}{l}\text { Word List Delayed } \\
\text { Recall }\end{array}$ & $0.1(-0.5,0.7)$ & $0.1(-0.5,0.8$ & 0.88 \\
\hline Trails A & $0.6(-5.4,6.6$ & $-1.2(-7.5,5.1$ & 0.21 \\
\hline Trails B & $-11.5(-23.3,0.3)$ & $-14.1(-26.5,-1.6)$ & 0.37 \\
\hline Digit Symbol & $2.2(-1.7,6.1)$ & $0.1(-4.0,4.2$ & 0.03 \\
\hline Block Design & $-0.5(-2.1,1.2)$ & $0.1(-1.6,1.8)$ & 0.15 \\
\hline
\end{tabular}

* ((last test - baseline test)/years between tests); adjusted for age at baseline, sex, race, education, hypertension at baseline, diabetes at baseline, eGFR, time on treatment, number of testing time-points, FAVORIT site, and baseline cognitive score. Mean follow up (95\% CI): Treatment, 3.3 years $(3.1,3.5)$; Control, 3.2 years $(3.0,3.4) ; \mathrm{P}>0.05$.

\section{Effect Modification by B-vitamin status}

Since nearly all participants had sufficient baseline vitamin B12 and folate concentrations and all had elevated tHcy, median splits were used to designate high and low status for tHcy and folate, and a cut-point of $<350 \mathrm{pg} / \mathrm{ml}$ was used for vitamin B12. A cut-point of $<30 \mathrm{nmol} / \mathrm{L}$ was considered indicative of Vitamin B6 insufficiency $(40,41)$. No interaction between baseline plasma concentrations and treatment efficacy for folate, vitamin B12, vitamin B6, or tHcy was found (data not shown). In addition, participants in the treatment group whose plasma tHcy concentrations were lowered below target $(<12 \mu \mathrm{mol} / \mathrm{L}$ for men, and $<11 \mu \mathrm{mol} / \mathrm{L}$ for women $)$ did not have better end-of-trial cognitive performance than did the high-dose participants whose tHcy was not lowered below target concentrations (data not shown).

\section{Discussion}

Among prevalent kidney transplant patients, a population with high tHcy levels and at high risk of cognitive impairment, high dose B-vitamin therapy to lower tHcy had a modest but statistically significant beneficial effect on cognitive function. With 548 participants from 18 centers in the US and Canada, this ancillary study to the FAVORIT trial is one of the largest trials assessing whether B-vitamin therapy to lower tHcy benefits cognitive function. It is also the largest longitudinal study of cognitive function in kidney transplant recipients to date.

The selective improvement in episodic memory, attention and processing speed among treated patients is noteworthy. By apriori design, we selected tests that reflect discrete aspects of cognitive function and analyzed the effect of treatment on each one. We did so because the different aspects of cognition that are probed by each test have shown different associations with homocysteine and each of the three B-vitamins, suggesting that different mechanisms might link these biomarkers to specific impairments in the context of different neurological conditions (eg. (2, 6, 42-49)). Here, as demonstrated by the percent impaired on the different cognitive tests, we found that the predominant cognitive impairment among kidney transplant recipients was on tests of executive function (Trails A\&B), and processing speed and attention (Digit Symbol Coding), whereas memory was affected to a lesser extent. This pattern of impairment is frequently seen in other cohorts with kidney disease $(23,24)$, and is reminiscent of Vascular Cognitive Impairment (50). The longitudinal improvement in processing speed and attention (Digit Symbol Coding) among those who received high-dose B-vitamin therapy is similar to the improvement in processing speed and memory in the treatment arm of the FACIT trial (18). Such an effect would be consistent with the prevention of cortical damage by B-vitamin therapy as observed in the VITACOG trial (20), and in a similar recent intervention $(51,52)$, and which is associated with decline in executive function as recently described by Zhang (53). Our findings suggest that these aspects of cognitive impairment might be partially reversible among kidney transplant recipients, and that at least attention and processing speed be amenable to further improvement through vitamin therapy. Thus in contrast to the controversial inference drawn from metaanalyses that B-vitamins do not benefit cognition (8-11), our findings provide evidence that B-vitamin therapy to 
lower tHcy can also have measurable cognitive benefit, if not for all populations, then at least in some clinically important conditions.

Although B-vitamin treatment significantly improved cognition in our cohort, the effect was modest, limited to specific domains, and its clinical implications remain undefined. Our cohort was selected for elevated plasma tHcy concentrations without regard for B-vitamin sufficiency and our primary objective was to determine whether an intervention with high-dose B-vitamins to lower tHcy would also benefit cognition as compared to placebo. The failure to normalize tHcy in nearly half of the treatment group, and the smaller but significant reduction in tHcy in the placebo group may have weakened any tHcy-related cognitive effects of treatment. In a randomized controlled trial of tHcy-lowering in individuals with MCI, de Jaeger and colleagues (19) found a significant benefit of B-vitamin treatment among participants with baseline tHcy above 11.3 micromol/L (the median for their study) in global cognitive function, episodic memory, and verbal fluency. It is possible the present study showed a smaller benefit from B-vitamin treatment because tHcy was not sufficiently lowered; however, in a subgroup analysis of our trial, participants whose tHcy concentrations were lowered "to target" did not have better cognitive function at follow-up than those whose plasma tHcy remained above this level (i.e. the study entry criteria of $12 \mu \mathrm{mol} / \mathrm{L}$ for men and 11 $\mu \mathrm{mol} / \mathrm{L}$ for women). Moreover, the degree of reduction in $\mathrm{tHcy}$ on treatment was not related to the extent of change in cognitive function in the subset of participants for whom these data were available. It is important to note, however, that almost none of the participants in this study were insufficient in folate or vitamin B12. It is possible that if this population had had a greater prevalence of folate and vitamin B12 deficiency, we would have observed a stronger response to treatment.

The strengths of our study include the double blind, placebo-controlled randomized trial design, carried out in a large, well-defined, patient population from multiple clinical centers; the relatively long mean follow-up, and the detailed neuropsychological testing. Consistent with the results of other studies of cognition in kidney transplant recipients (54), we found a high prevalence of cognitive impairment in our cohort both at baseline (29) and follow-up. Contrary to our prediction, however, cognition did not decline significantly over the course of the study in either study arm. The lack of decline may reflect the lower than expected rate of incident and recurrent cardiovascular events in the FAVORIT parent trial population (30), suggesting potential selection bias in the trial population for better health and/or clinicalcare than is typical for the general population of kidney transplant recipients.

The lower than expected event-rate may be one reason that the parent FAVORIT trial failed to show efficacy for the primary cardiovascular endpoints (30). Nevertheless, we found modest but statistically significant cognitive improvement and benefit of B-vitamin treatment cognitive tests relating to executive function and shortterm verbal memory. The results may be limited by the fact that the control group also received a multi-vitamin containing vitamins B12 and B6 (but not folic acid), albeit at a much lower dose. Our finding that high dose B-vitamin treatment benefited these cognitive outcomes despite a null finding on the primary cardiovascular end-point is reminiscent of the results of the FACIT trial (Folic Acid for Carotid Intima Thickness), where folic acid treatment improved cognition compared to control, despite no difference between treatment and control for the trial's primary cardiovascular outcome (18).

A second limitation of the study is that we had relatively few participants for whom we were able to administer baseline cognitive testing prior to randomization into the parent trial and initiation of the intervention. This limited our analyses to primarily crosssectional end-of-trial group comparisons rather than comparison of change scores. We did include, however, longitudinal analyses in the 131 participants for whom these data were available.

Other considerations arguably restrict the generalizability of our findings to other populations. For example, the average age in our cohort was younger than in most studies linking tHcy to cognitive decline. This might have contributed to the fact that we did not observe the cognitive decline that is associated with hyperhomocysteinemia in older populations (55). Furthermore, kidney transplant recipients can carry a considerable burden of preexisting and potentially irreversible neurological damage related to their prior end stage renal disease (ESRD), previous effects of dialysis, and current immunosuppressive medication, and persistent morbidity such as hypertension and diabetes. Although cognitive performance typically improves after transplantation, persisting cognitive impairment is common $(54,56-58)$. While relatively subtle, an impairment of 1 or more standard deviations below the norm of any cognitive test can be considered to be clinically meaningful and may increase the risk of further deterioration over time. The observation of a modest improvement in the treatment arm against this background of pre-existing cognitive impairment and co-morbidity, suggests there may be room for further improvement in this patient population.

In conclusion, high-dose B-vitamin supplementation provided modest benefit for some cognitive functions in our cohort of kidney transplant recipients. While cognitive impairment was common among our participants, progressive decline was unexpectedly rare over the course of the study. This may have limited our ability to evaluate the effects of tHcy lowering on cognition more fully. It is possible that chronically high tHcy is a risk factor for cerebrovascular pathology and cognitive impairment, but that damage from long-term 
exposure is difficult to reverse. This may be especially relevant to our participants since plasma tHcy is significantly elevated in patients with chronic kidney disease. The question of potential cognitive benefits of B-vitamin therapy in individuals with inadequate B-vitamin status remains open and highlights the need to identify other metabolic derangements that determine the extent of cognitive benefit under B-vitamin therapy.

Funding: This research was supported by grant RO1 DK65114 and cooperative agreement UO1 DK61700 from the from the National Institute of Diabetes and Digestive and Kidney Diseases with additional financial support from the Office of Dietary Supplements, National Institutes of Health, Department of Health and Human Services, and by cooperative research agreement 58-1950-4-401 with the U.S. Department of Agriculture. Partial support to AMT was provided by Israel Science Foundation grant no. 1353/11 and a European Union FP7 Marie Curie International Re-integration grant PIRG08-GA-2010-276791 - NUVASCOG.

Conflict of Interest Disclosure: Jacob Selhub has consulted for Pam Lab, Inc. None of the other authors have conflicts to disclose.

Acknowledgements: We are grateful to the FACT study coordinators Dr. Kristen E. D'Anci and Ms. Beverly Dobson for their tireless efforts, to Gerard E. Dalla for statistical consultation, to the FACT study investigators and nurses at all of the 18 participating FAVORIT sites, to Andrew S. Levey and the FAVORIT study executive committee, and to each of the volunteers, without whom the study would not have been possible.

Ethical standards: The study received approval from the institutional review or ethics boards of all 18 clinical sites and Tufts University. Written informed consent was obtained from all participants.

\section{References}

1. Selhub, J., et al., B vitamins, homocysteine, and neurocognitive function in the elderly. Am J Clin Nutr, 2000. 71(2): p. 614S-620S.

2. Riggs, K.M., et al., Relations of vitamin B-12, vitamin B-6, folate, and homocysteine to cognitive performance in the Normative Aging Study. Am J Clin Nutr, 1996. 63(3): p. 306-14.

3. Troen, A. and I. Rosenberg, Homocysteine and cognitive function. Semin Vasc Med, 2005. 5(2): p. 209-14.

4. Bertsch, T., et al., Homocysteine in cerebrovascular disease: an independen risk factor for subcortical vascular encephalopathy. Clin Chem Lab Med, 2001. 39(8): p. 721-4.

5. Sachdev, P., Homocysteine, cerebrovascular disease and brain atrophy. J Neurol Sci, 2004. 226(1-2): p. 25-9.

6. Kado, D.M., et al., Homocysteine versus the vitamins folate, B6, and B12 as predictors of cognitive function and decline in older high-functioning adults: MacArthur Studies of Successful Aging. Am J Med, 2005. 118(2): p. 161-7.

7. Clarke, R., et al., Folate, vitamin B12, and serum total homocysteine levels in confirmed Alzheimer disease. Arch Neurol, 1998. 55(11): p. 1449-55.

8. Clarke, R., et al., Effects of homocysteine lowering with B vitamins on cognitive aging: meta-analysis of 11 trials with cognitive data on 22,000 individuals. Am J Clin Nutr, 2014. 100(2): p. 657-66.

9. McCaddon, A. and J.W. Miller, Assessing the association between homocysteine and cognition: reflections on Bradford Hill, meta-analyses, and causality. Nutr Rev, 2015. 73(10): p. 723-35.

10. Nachum-Biala, Y. and A.M. Troen, B-vitamins for neuroprotection: narrowing the evidence gap. Biofactors, 2012. 38(2): p. 145-50.

11. Smith, A.D., et al., Homocysteine lowering, B vitamins, and cognitive aging. Am J Clin Nutr, 2015. 101(2): p. 415-6.

12. McMahon, J.A., et al., A controlled trial of homocysteine lowering and cognitive performance. New England Journal of Medicine, 2006. 354(26): p. 2764-72.

13. Stott, D.J., et al., Randomized controlled trial of homocysteine-lowering vitamin treatment in elderly patients with vascular disease. Am J Clin Nutr, 2005. 82(6): p. 1320-6.

14. van Uffelen, J.G., et al., Walking or vitamin B for cognition in older adults with mild cognitive impairment? A randomised controlled trial. Br J Sports Med, 2008. 42(5): p. 344-51.

15. Eussen, S.J., et al., Effect of oral vitamin B-12 with or without folic acid on cognitive function in older people with mild vitamin B-12 deficiency: a randomized, placebo-controlled trial. Am J Clin Nutr, 2006. 84(2): p. 361-70.

16. Sommer, B.R., A.L. Hoff, and M. Costa, Folic acid supplementation in dementia: a preliminary report. J Geriatr Psychiatry Neurol, 2003. 16(3): p. 156-9.

17. Nilsson, K., L. Gustafson, and B. Hultberg, Improvement of cognitive functions after cobalamin/folate supplementation in elderly patients with dementia and elevated plasma homocysteine. Int J Geriatr Psychiatry, 2001. 16(6): p. 609-14

18. Durga, J., et al., Effect of 3-year folic acid supplementation on cognitive function in older adults in the FACIT trial: a randomised, double blind, controlled trial. Lancet, 2007. 369(9557): p. 208-16.

19. de Jager, C.A., et al., Cognitive and clinical outcomes of homocysteinelowering B-vitamin treatment in mild cognitive impairment: a randomized controlled trial. Int J Geriatr Psychiatry, 2012. 27(6): p. 592-600.

20. Smith, A.D., et al., Homocysteine-lowering by B vitamins slows the rate of accelerated brain atrophy in mild cognitive impairment: a randomized controlled trial. PLoS One, 2010. 5(9): p. e12244.

21. Douaud, G., et al., Preventing Alzheimer's disease-related gray matter atrophy by B-vitamin treatment. Proc Natl Acad Sci U S A, 2013. 110(23): p. 9523-8.

22. Bostom, A.G., et al., Elevated fasting total plasma homocysteine levels and cardiovascular disease outcomes in maintenance dialysis patients. A prospective study. Arterioscler Thromb Vasc Biol, 1997. 17(11): p. 2554-8.

23. Sarnak, M.J., et al., Frequency of and risk factors for poor cognitive performance in hemodialysis patients. Neurology, 2013. 80(5): p. 471-80.

24. Weiner, D.E., et al., Albuminuria, cognitive functioning, and white matter hyperintensities in homebound elders. Am J Kidney Dis, 2009. 53(3): p. 438 47.

25. Friedman, A.N., et al., Hyperhomocysteinemia in renal transplant recipients. Am J Transplant, 2002. 2(4): p. 308-13.

26. Bostom, A.G., et al., Prevalence of mild fasting hyperhomocysteinemia in renal transplant versus coronary artery disease patients after fortification of cereal grain flour with folic acid. Atherosclerosis, 1999. 145(1): p. 221-4.

27. Bostom, A.G., et al., Treatment of hyperhomocysteinemia in renal transplant recipients. A randomized, placebo-controlled trial. Ann Intern Med, 1997 127(12): p. 1089-92.

28. Beaulieu, A.J., et al., Enhanced reduction of fasting total homocysteine levels with supraphysiological versus standard multivitamin dose folic acid supplementation in renal transplant recipients. Arterioscler Thromb Vasc Biol, 1999. 19(12): p. 2918-21.

29. Troen, A.M., et al., Cognitive dysfunction and depression in adult kidney transplant recipients: baseline findings from the FAVORIT Ancillary Cognitive Trial (FACT). J Ren Nutr, 2012. 22(2): p. 268-76 e1-3.

30. Bostom, A.G., et al., Homocysteine-lowering and cardiovascular disease outcomes in kidney transplant recipients: primary results from the Folic Acid for Vascular Outcome Reduction in Transplantation trial. Circulation, 2011. 123(16): p. 1763-70.

31. Bostom, A.G., et al., Rationale and design of the Folic Acid for Vascular Outcome Reduction In Transplantation (FAVORIT) trial. Am Heart J, 2006. 152(3): p. 448 e1-7.

32. Araki, A and Y. Sako, Determination of free and total homocysteine in human plasma by high-performance liquid chromatography with fluorescence detection. J Chromatogr, 1987. 422: p. 43-52.

33. Levey, A.S., et al., A new equation to estimate glomerular filtration rate. Ann Intern Med, 2009. 150(9): p. 604-12.

34. Shin, Y.S., et al., Pyridoxal-5'-phosphate determination by a sensitive micromethod in human blood, urine and tissues; its relation to cystathioninuria in neuroblastoma and biliary atresia. Clin Chim Acta, 1983 127(1): p. 77-85.

35. Wechsler, D., Manual for the Wechsler Memory Scale. 3rd ed. 1997, San Antonio, TX: Psychological Corporation.

36. Wechsler, D., Wechsler Adult Intelligence Scale. 3rd ed. 1997, San Antonio, TX: The Psychological Corporation/Harcourt Brace and Company.

37. Heaton, R.K., I. Grant, and e. al., Comprehensive Norms for an Expanded Halstead-Reitan Battery. 1991, Odessa, FL: Psychological Assessment Resources, Inc.

38. Savage, D.G., et al., Sensitivity of serum methylmalonic acid and total homocysteine determinations for diagnosing cobalamin and folate deficiencies. Am J Med, 1994. 96(3): p. 239-46.

39. Carmel, R., et al., Update on cobalamin, folate, and homocysteine Hematology Am Soc Hematol Educ Program, 2003: p. 62-81.

40. Leklem, J.E., Vitamin B-6: a status report. J Nutr, 1990. 120 Suppl 11: p. $1503-$ 7.

41. Sakakeeny, L., et al., Plasma pyridoxal-5-phosphate is inversely associated with systemic markers of inflammation in a population of U.S. adults. J Nutr, 2012. 142(7): p. 1280-5.

42. Feng, L., et al., Homocysteine, folate, and vitamin B-12 and cognitive performance in older Chinese adults: findings from the Singapore Longitudinal Ageing Study. Am J Clin Nutr, 2006. 84(6): p. 1506-12.

43. Moorthy, D., et al., Status of vitamins B-12 and B-6 but not of folate, homocysteine, and the methylenetetrahydrofolate reductase C677T polymorphism are associated with impaired cognition and depression in adults. J Nutr, 2012. 142(8): p. 1554-60.

44. Parizkova, M., et al., Homocysteine and Real-Space Navigation Performance among Non-Demented Older Adults. J Alzheimers Dis, 2017. 55(3): p. 951964.

45. Philip, D., et al., Dihydrofolate reductase 19-bp deletion polymorphism modifies the association of folate status with memory in a cross-sectional 
multi-ethnic study of adults. Am J Clin Nutr, 2015. 102(5): p. 1279-88.

46. Ramos, M.I., et al., Plasma folate concentrations are associated with depressive symptoms in elderly Latina women despite folic acid fortification. Am J Clin Nutr, 2004. 80(4): p. 1024-8.

47. Dufouil, C., et al., Homocysteine, white matter hyperintensities, and cognition in healthy elderly people. Ann Neurol, 2003. 53(2): p. 214-21.

48. Elkins, J.S., et al., Methylenetetrahydrofolate reductase C677T polymorphism and cognitive function in older women. Am J Epidemiol, 2007. 166(6): p. 6728.

49. Hsu, W.C., et al., Digit symbol substitution test score and hyperhomocysteinemia in older adults. Medicine (Baltimore), 2016. 95(35): p. e4535.

50. O'Brien, J.T., Vascular cognitive impairment. Am J Geriatr Psychiatry, 2006. 14(9): p. 724-33.

51. Hara, J., et al., Novel Therapy of Hyperhomocysteinemia in Mild Cognitive Impairment, Alzheimer's Disease, and Other Dementing Disorders. J Nutr Health Aging, 2016. 20(8): p. 825-834.

52. Shankle, W.R., et al., CerefolinNAC Therapy of Hyperhomocysteinemia Delays Cortical and White Matter Atrophy in Alzheimer's Disease and Cerebrovascular Disease. J Alzheimers Dis, 2016. 54(3): p. 1073-1084.
53. Zhang, X., et al., Bayesian model reveals latent atrophy factors with dissociable cognitive trajectories in Alzheimer's disease. Proc Natl Acad Sci U S A, 2016. 113(42): p. E6535-E6544.

54. Van Sandwijk, M.S., et al., Cognitive Changes in Chronic Kidney Disease and After Transplantation. Transplantation, 2016. 100(4): p. 734-42.

55. Elias, M.F., et al., Homocysteine and cognitive performance in the Framingham offspring study: age is important. Am J Epidemiol, 2005. 162(7): p. 644-53.

56. Gelb, S., et al., Cognitive outcome following kidney transplantation. Nephrol Dial Transplant, 2008. 23(3): p. 1032-8.

57. Griva, K., et al., Neuropsychological performance after kidney transplantation: a comparison between transplant types and in relation to dialysis and normative data. Nephrol Dial Transplant, 2004. 19(7): p. 1866-74.

58. Harciarek, M., et al., Cognitive performance before and after kidney transplantation: a prospective controlled study of adequately dialyzed patients with end-stage renal disease. J Int Neuropsychol Soc, 2009. 15(5): p. 684-94.

59. Radloff, L., The CES-D scale: a self-report depression scale for research in the general population. . Appl Psychologic Measurem, 1977. 1: p. 385-491. 